JOURNAL OF

SYMPLECTIC GEOMETRY

Volume 5, Number 1, 2007

\title{
DEDICATION TO DUSA MCDUFF
}

In the late 1960's V.I. Arnold posed a question which preoccupied geometers for the next two decades: Does global symplectic geometry exist? Some work by Weinstein and Moser in the 1970's suggested that the answer to this question might be negative, but a decade later Gromov's theory of pseudoholomorphic curves and other innovative techniques that he invented (symplectic blowing-up, symplectic branched coverings,...) made it clear that the answer was a resounding yes. Dusa McDuff was among the first persons to realize the power of these new methods. Beginning with her papers in the mid and late eighties (Symplectic diffeomorphisms and the flux homomorphism, Examples of simply-connected symplectic non-Käehlerian manifolds, Examples of symplectic structures, Elliptic methods in symplectic geometry,...) and in a long series of subsequent papers on these Gromov themes (among them the seminal papers Symplectic packings and algebraic geometry with Leonid Polterovich, and The classification of ruled symplectic 4-manifolds with François Lalonde), she has made an overwhelming case for the efficacy of this new technology. In addition her two books coauthored with Dietmar Salamon have by now become the standard references for all graduate students who want to learn about modern symplectic geometry or master the tools of "J-holomorphic" analysis.

This is the first of two special issues of JSG honoring Dusa's achievements. As a quick survey of the papers in this volume makes vividly clear, the techniques which she has done so much to bring into the mainstream of modern-day symplectic geometry are playing an ever-expanding and ever more indispensable role.

the Editors 